Article

\title{
Evaluation of Rate Adaptation Algorithms in IEEE 802.11 Networks
}

\author{
Ibrahim Sammour *,+(D) and Gerard Chalhoub + \\ LIMOS, University of Clermont-Auvergne, 63170 Aubière, France; gerard.chalhoub@uca.fr \\ * Correspondence: ibrahim.sammour@uca.fr; Tel.: +33-6-5932-5635 \\ + These authors contributed equally to this work.
}

Received: 31 July 2020; Accepted: 29 August 2020; Published: 3 September 2020

\begin{abstract}
Wireless technologies are being used in various applications for their ease of deployment and inherent capabilities to support mobility. Most wireless standards supports multiple data rates that may vary between few Mbps to few Gbps. Reaching the maximum supported data rate is what most application seek for. Nevertheless, the choice of data rates is very closely related to the quality of communication links and their stability. IEEE 802.11 standard introduced multi-rate support, since then, a lot of research has been done on rate adaptation, dealing with the different parameters that lead to an estimation of the channel conditions and the metrics that affect the network performance. In this paper, we present some of the popular rate adaptation schemes and summarize their characteristics. We categorize them as well into different categories according to their design and functionalities in terms of the strategies that are used to estimate channel conditions and decision making. We implemented some algorithms from the different categories in the network simulator NS-3 in order to evaluate their performance under different scenarios in Ad hoc and infrastructure modes. We present the lessons learned as well as our insights for future research work that can enhance the current approaches in the literature.
\end{abstract}

Keywords: rate adaptation algorithms; wifi; IEEE 802.11; mobility; interference

\section{Introduction}

Wireless communication technologies are a very attractive solution for establishing connectivity between mobile nodes with minimal cable deployment. Many applications rely on such technologies in order to operate such as C-ITS (Cooperative-Intelligent Transport Systems) applications for vehicular networks [1,2], precision farming with mobile connected engines [3,4], the wide variety of applications used on mobile phones using cellular networks. Most of these applications require the support of mobile nodes while maximizing the throughput. Maximum throughput is achieved when the communication technology is able to reach its maximum possible physical data rates. The communication links should be of very good quality and stable in order to reach these data rates. This is a very challenging aspect to guarantee under mobility and in the presence of interference. Indeed, mobility will have a negative impact on the Received Signal Strength (RSS) when the distance between the transmitter and the receiver increases, and interference will decrease the Signal to Interference plus Noise Ratio (SINR). Hence, most communication technologies adopt dynamic rate adaptation algorithms in order to choose the best possible data rate that allows the establishment of communications [5-8].

Rate Adaptation is one of the key aspects of the functionalities of IEEE 802.11's physical layer. It works by assessing the channel conditions and taking a decision to adapt the transmission rate by selecting a combination of transmission features, such as the modulation and coding schemes (MCS), guard interval, and channel width. These features can be selected depending on the version 
of the standard being used. When the signal is strong when compared to the level of noise and interference, an algorithm can select a high transmission rate, which leads to less occupancy of the channel and higher throughput. On the other hand, when the signal is weak as compared to the level of noise and interference, a lower transmission rate will ensure the delivery of the packets, but leads to more occupancy time. A lot of factors affect the decision making, such as node mobility, interference, and channel fading.

Selecting the appropriate transmission rate after estimating channel conditions is an important task in improving the performance of wireless links. Rate adaptation schemes rely on certain metrics, such as Frame Loss Ratio (FLR) and SINR. A good rate adaptation scheme is defined by its responsiveness to fast channel changes and achieving the highest possible throughput. Rate adaptation schemes have been an active research topic for about two decades, many algorithms have been proposed in the literature. Very few of them have been tested in fully mobile IEEE 802.11 networks. The primary contributions of this paper are (i) categorization of rate adaptation schemes proposed in the literature, (ii) evaluation through simulation of the performance of four different rate adaptation algorithms under different scenarios highlighting the impact of mobility and interference in Ad hoc and infrastructure modes using NS-3 simulator, and (iii) presenting the lessons learned as well as our insights for future research work that can enhance existing approaches.

The rest of the paper is organized, as follows. In Section 2, we list and categorize several rate adaptation schemes explaining the algorithms focusing on how the channel conditions are estimated and their decision making. In Section 3, we present our simulation scenarios and analyze the obtained results. In Section 4, we present the lessons learned as well as our insights for future research work on rate adaption techniques. Finally, we conclude the paper in Section 5.

\section{Related Work}

In this section, we will present a brief summary of the main characteristics of existing rate adaptation algorithms (RAA). RAAs can be classified into various categories according to the metrics that they use to evaluate the channel or link quality such as frame loss and SNR in [9], or consecutive transmission count, frame loss ratio, transmission time, throughput, SNR, bit error rate, and combined metrics in [10]. We chose to classify RAAs into three categories: (i) Explicit Feedback, in which RAAs base their bit rate adaptation on the receiver feedback, (ii) Implicit Feedback, in which RAAs base their adaptation on the information available on the sender side, and (iii) Hybrid, which is a category that combines information from feedback of the receiver and information available to the sender. In what follows we will describe each of these categories by selecting representative RAAs of each category. Table 1 summarizes the information about the different RAA algorithms presented in this section.

\subsection{Explicit Feedback}

Explicit Feedback is known as a receiver-driven rate adaptation scheme where the receiver takes a decision that is based on its estimation of the channel conditions and relays it back to the sender via different approaches using control frames, such as Clear To Send (CTS) and Acknowledgements (ACK).

On Demand Feedback Rate Adaptation (OFRA) [11] is a receiver based rate adaptation algorithm (RAA), where the channel quality is estimated at the receiver based on SINR values. The receiver selects the optimal bit rate from a lookup table created previously. It contains a set of thresholds at which data rates should be changed. This information is fed back to the sender on demand while using ACK frames. In case of an ACK-less traffic, OFRA uses a specially designed feedback frame. OFRA presents some limitations, such as modifying the ACK frame that is a violation of the standard, introduction of additional overhead with the special feedback frame, which is sent at the lowest data rate.

SINR-aware Intra-frame Rate Adaptation (SIRA) [12] selects two rates for a single Aggregate MAC Protocol Data Unit (A-MPDU) transmission. It finds the starting symbol "I" when the rate should be changed. When the condition $S I N R_{i}<S I N R_{t h}$ is met, the symbol "I" is found. SINR $R_{t h}$ is 
the minimum SINR at which the theoretical Bit Error Rate (BER) of the primary rate is less than $10^{-4}$. Subsequently, "I" is fed back to the sender via the BlockAck. The main drawback of SIRA is that it only determines two rates for an aggregated frame, which may not be enough for a fast-changing channels.

An Ideal RAA is implemented in the famous network simulator NS-3. This RAA initially creates a table of SINR and MCS pairs. The SINR thresholds in this table ensure selecting an MCS that leads to a BER below a certain value. For example, the default value is $10^{-5}$ ), the SINR is fed back from the receiver to the transmitter via a perfect out-of-band mechanism. The main drawback of this mechanism is the use of an out-of-band channel for sending back the feedback which is not available in Industrial, Scientific, and Medical (ISM) bands used by IEEE 802.11.

Table 1. Summary of existing rate adaptation algorithms.

\begin{tabular}{|c|c|c|c|c|c|}
\hline Name & Category & Metrics & Pros & Cons & $\begin{array}{l}\text { Modify } \\
\text { Standard }\end{array}$ \\
\hline Ideal & $\begin{array}{l}\text { Explicit } \\
\text { Feedback }\end{array}$ & SINR & $\begin{array}{l}\text { Instantaneous feedback and } \\
\text { rate decision }\end{array}$ & $\begin{array}{l}\text { Uses perfect out-of-band } \\
\text { mechanism for feedback }\end{array}$ & Yes \\
\hline OFRA & $\begin{array}{l}\text { Explicit } \\
\text { Feedback }\end{array}$ & SINR & $\begin{array}{l}\text { Feedback is sent only when a } \\
\text { new rate is selected }\end{array}$ & $\begin{array}{l}\text { Additional frame is sent for } \\
\text { feedback at the lowest rate }\end{array}$ & Yes \\
\hline SIRA & $\begin{array}{l}\text { Explicit } \\
\text { Feedback }\end{array}$ & SINR & $\begin{array}{l}\text { Uses } 2 \text { rates for a single } \\
\text { A-MPDU transmission to } \\
\text { adapt with channel changes }\end{array}$ & $\begin{array}{l}\text { In a fast-changing channel } \\
\text { we may need more than } 2 \\
\text { rates for a single A-MPDU } \\
\text { transmission to adopt to } \\
\text { channel changes }\end{array}$ & No \\
\hline Strale & $\begin{array}{l}\text { Implicit } \\
\text { Feedback }\end{array}$ & Throughput & $\begin{array}{l}\text { Prefers to use an optimal } \\
\text { A-MPDU length instead of } \\
\text { decreasing the rate }\end{array}$ & $\begin{array}{l}\text { Does not take interference } \\
\text { into consideration }\end{array}$ & No \\
\hline Minstrel-HT & $\begin{array}{l}\text { Implicit } \\
\text { Feedback }\end{array}$ & Throughput & $\begin{array}{l}\text { It can select a high rate with } \\
\text { a significant FLR as long } \\
\text { as it can achieve the best } \\
\text { throughput }\end{array}$ & $\begin{array}{l}\text { In some conditions it fails to } \\
\text { enhance the throughput [13] }\end{array}$ & No \\
\hline MiRA & $\begin{array}{l}\text { Implicit } \\
\text { Feedback }\end{array}$ & Throughput & $\begin{array}{l}\text { Collision-based decision } \\
\text { making and probing interval } \\
\text { adaptation }\end{array}$ & $\begin{array}{l}\text { Introduces overhead } \\
\text { when using the RTS/CTS } \\
\text { mechanism }\end{array}$ & No \\
\hline RAMAS & $\begin{array}{l}\text { Implicit } \\
\text { Feedback }\end{array}$ & PLR & $\begin{array}{l}\text { It is efficient in MIMO where } \\
\text { it selects between different } \\
\text { values of transmission } \\
\text { metrics }\end{array}$ & $\begin{array}{l}\text { Slow in adapting the number } \\
\text { of streams and premature } \\
\text { MCS adaptation }\end{array}$ & No \\
\hline Damysus & $\begin{array}{l}\text { Implicit } \\
\text { Feedback }\end{array}$ & PLR & $\begin{array}{ll}\text { Performs well in } & \text { in } \\
\text { dense environments } & \text { in } \\
\text { infrastructure mode } & \\
\end{array}$ & $\begin{array}{l}\text { Is not applicable in Ad Hoc } \\
\text { mode }\end{array}$ & No \\
\hline MutFed & Hybrid & SINR,FLR & $\begin{array}{l}\text { Distinguishes the cause of } \\
\text { frame loss combined with } \\
\text { SINR decision }\end{array}$ & $\begin{array}{l}\text { In a dense environment } \\
\text { many frame losses cause the } \\
\text { algorithm to send at lower } \\
\text { rates }\end{array}$ & Yes \\
\hline EasiRA & Hybrid & ESS,PLR & $\begin{array}{l}\text { Combines external } \\
\text { sensors and ESS to detect } \\
\text { collisions and uses this } \\
\text { information with the PLR for } \\
\text { rate selection }\end{array}$ & $\begin{array}{l}\text { It tries to identify collisions } \\
\text { using external information, } \\
\text { such as sensor-hints and ESS } \\
\text { which may not be available } \\
\text { in all the devices }\end{array}$ & No \\
\hline
\end{tabular}

\subsection{Implicit Feedback}

Implicit Feedback is a sender-driven rate adaptation scheme and it is usually based on Packet Error Rate (PER). The main idea is for the sender to select an appropriate data rate that is based on the PER observed on his side. This mainly requires ACK to be used to enable the sender to calculate PER.

STRALE [14] jointly adapts PHY rate and A-MPDU length. After receiving a BlockAck, it calculates the optimal A-MPDU length for the highest throughput of the last A-MPDU transmission. Subsequently, the next A-MPDU length of the next transmission is determined using the Exponential 
Weighted Moving Average (EWMA). The difference between the latest and the newly calculated A-MPDU length is compared to a certain threshold. If it is greater than the threshold, STRALE takes the decision on Modulation and Coding Scheme (MCS) and A-MPDU length after checking if the throughput with one lower MCS index using the latest A-MPDU length is better than staying at the same MCS index with the newly calculated A-MPDU length. The main drawback of Strale is that it does not take interference into consideration.

Minstrel-HT [15] relies on three parameters: channel width, guard interval, and number of streams to create group rates. Each group contains eight different data rates represented by the MCS index. Minstrel uses probing to determine the best data rate. This process consists of two periods: sampling and non-sampling periods. During the sampling period, Minstrel-HT selects a random data rate from all of the available data rates in each group. If a data rate results in a higher throughput than the previous data rate, then it is used for subsequent MPDU transmissions. Otherwise, Minstrel-HT keeps using the previous data rate. Throughput is calculated based on Frame Loss Rate (FLR) while taking EWMA into account. At the end of the sampling period, Minstrel-HT collects three data rate options: best throughput, second best throughput, and best probability. Subsequently, in the non-sampling period, MPDUs are sent using the best data rate until the maximum number of retransmissions is reached in the case of packet loss then the second best data rate is used. Similarly, if the second best data rate experiences packet loss, the best probability data rate is used. The evaluation in [13] showed that in some conditions Minstrel-HT fails to enhance throughput, especially in a non-fading channel when the quality of the channel changes back from bad to good.

MiRA [16] is a rate adaptation that is used for Multiple-Input and Multiple-Output (MIMO) channels. It overcomes MPDU loss by applying a zigzag rate adaptation between intra-mode and inter-mode. MiRA first performs probing on the rate in MIMO intra-mode. In case goodput fails to be increased in intra-mode, MiRA zigzags to inter-mode MIMO, the probing mechanism only starts if significant changes occur in the measured moving average goodput of the current rate. The probing interval of MiRA is also adapted, which limits the probing number when goodput is low. MiRA also considers frame aggregation and Block Acknowledgement schemes when performing the best data rate probing. It also includes a collision aware mechanism where the sender detects collision if it satisfies the condition that the aggregate frame has experienced at least one retry and the loss ratio of its sub-frames is less than $10 \%$. If collision exists, it triggers the adaptive RTS/CTS mechanism. The main drawback of MiRA is the introduction of an overhead when using the RTS/CTS mechanism.

RAMAS [17] is a credit based approach. The data rates are grouped into two groups: modulation and enhancement groups. The modulation group consists of the different MCS values. The enhancement group consists of spatial stream, guard interval, and channel width. RAMAS uses credit-based algorithms, which relies on the packets success and failure statistics, to adapt these groups independently of each other and combines the results together in order to decide the overall feature setting. In each group, different rules are applied to increase or decrease the data rate sequentially. The main drawback of RAMAS is that it performs poorly, because its credit based scheme is conservative in adapting the number of streams and aggressive in adapting the MCS. This mismatch causes RAMAS to often operate at suboptimal settings with single stream and high MCS values leading to higher losses and reduced performance, as shown in the evaluations in [18].

Damysus [19] addresses 802.11ax exploiting the Basic Service Set (BSS) Color Scheme. It increases the transmission opportunities by using adaptive Overlapping Basic Service Set/Preamble-Detection (OBSS/PD) thresholds which leads to a higher contention level inside a BSS and jointly adjusts the transmit power level. A statistical study is done during an interval of $100 \mathrm{~ms}$ and during a cycle of $1 \mathrm{~s}$ were success and failure of packet transmissions are recorded and compared to the success and failure thresholds. It is then decided whether to increase or decrease either the rate, the OBSS/PD threshold, or the transmission power, depending on the statistical results collected. The main drawback of Damysus is relying on packet loss ratio thresholds. In [20] several experiments were done in order 
to verify that there is no single best Packet Loss Ratio (PLR) threshold that can help to achieve the maximum throughput.

\subsection{Hybrid Adaptation}

In Mutual Feedback (MutFed) [21], the SINR is measured on the receiver side. After 10 frames a new rate selection decision is made, either to stay at the same rate or to change the rate. This decision is made based on a table that maps SINR threshold ranges to rates. The newly selected rate is fed back to the sender by sending an ACK with the proposed rate. On the transmitter side, if two frame transmissions fails, then the transmitter automatically lowers the MCS for the following transmission. The main drawback of MutFed is not differentiating the result of packet loss that may result in lowering the rate when the packets are not lost due to the channel degradation.

EasiRA [22] measures the link quality by two means. First, it calculates the FLR and combines it with mobility and other sensor information. Secondly it obtains the environmental signal strength (ESS) information to help differentiate the causes of packet loss. When a packet cannot be successfully received due to bit errors, the receiver sends back a special control frame, named "non-acknowledgement NAK", to the transmitter in order to inform it that it may suffer collision. If the transmitter does not receive neither an ACK or a NAK then it reduces the rate. Finally, it combines the random and deterministic rate adaptation mechanisms together. The main drawback of EasiRA is the fact that it tries to identify collisions while using external information, such as sensor-hints and ESS, which may not be available in all of the devices.

\section{Performance Evaluation}

In this section, we selected representative algorithms from each of the categories in order to evaluate their performance under mobility scenarios. Very few papers have dealt with mobility and interference impact on RAAs, according to our knowledge. The selected algorithms are: Ideal, Strale, MinstrelHT, and MutFed. The selected algorithms cover the different categories, MinstrelHT and Strale are both implicit feedback algorithms, MinstrelHT, which is a widely used algorithm, as it is implemented in the linux kernel [23], claims being able to select a sampling rate that results in the highest throughput and highest probability of successful delivery of the frames. The main difference is that Strale can be used as an extension algorithm, it can be placed on top of MinstrelHT to enhance its performance, and it is a promising algorithm that tries to adapt the number of MPDUs in aggregated frames without reducing the transmission rate. To our knowledge, it has not been tested in a dense environment. Not many hybrid RAAs are available in the literature, we selected the MutFed scheme that is the most generic algorithm in its category compared to the other hybrid algorithms proposed for specific scenarios. The idea behind Mutfed is promising due to its ability to distinguish the reason of packet loss and the fact that it relies on SINR. We believe that these algorithms give a comprehensive understanding of RAAs due to the fact that they rely on a variety of different metrics and approaches that are commonly used in the RAAs in the literature. We used the network simulator NS-3 to compare and evaluate the performance of these algorithms. Ideal and Minstrel-Ht rate adaptation algorithms are already implemented in the simulator. We modified the original implementation of the Ideal algorithm in the simulator in order to ensure a fair comparison between the selected algorithms. Indeed, we consider that all of the nodes have only one communication channel for data and control traffic exchange. Hence, for Ideal, we included the feedback in the reserved bits of the BlockAck, which is sent back to the transmitter on the same communication channel.

We implemented the other two algorithms (Strale and MutFed). We chose the following metrics for the performance evaluation: (i) throughput, which is calculated at the physical layer, it gives a global view of the achieved performance of RAAs. (ii) Selected MCS, which indicates the MCS values selected by the nodes, it gives an idea about the different choices made by RAAs. Table 2 shows the different modulation and coding schemes of IEEE 802.11ac. (iii) FLR based on MPDUs, it is calculated based on the BlockAck information, it gives an idea about the overall lost MPDUs. (iv) FLR based on 
A-MPDUs, it is calculated based on the number of times the transmitter had to retransmit its frame, it gives an idea about the efficiency of RAAs in selecting an MCS that avoids loosing frames. Indeed, choosing a high MCS would cause A-MPDU frames to be lost at the receiver. This means that high A-MPDU frame loss means that the RAA is making a wrong decision in increasing the MCS.

All of these performance metrics are evaluated according to the number of nodes in the network. We increased the number of nodes while maintaining the same deployment surface. This means that increasing the number of nodes will increase the traffic load in the network and the interference level. This, in turn, will help us to evaluate the behavior of algorithms when faced with increased interference.

Table 2. Modulation and coding schemes.

\begin{tabular}{lll}
\hline MCS Index & Modulation Type & Coding Rate \\
\hline 0 & BPSK & $1 / 2$ \\
1 & QPSK & $1 / 2$ \\
2 & QPSK & $3 / 4$ \\
3 & 16-QAM & $1 / 2$ \\
4 & 16-QAM & $3 / 4$ \\
5 & 64-QAM & $2 / 3$ \\
6 & 64-QAM & $3 / 4$ \\
7 & 64-QAM & $5 / 6$ \\
8 & 256-QAM & $3 / 4$ \\
9 & 256-QAM & $5 / 6$ \\
\hline
\end{tabular}

All of the algorithms are evaluated in three different scenarios: (i) single link scenario, which is useful for highlighting the impact of link degradation due to mobility on RAAs. This allows us to evaluate the efficiency of RAAs is adapting the bit rate according to RSSI values only without interference. (ii) Infrastructure mode network, which represents the most widely used deployment mode. This scenario allows for us to evaluate the behavior of RAAs in a standard deployment with one Access Point through which all the network traffic should pass in order to be relayed to a wired network. In such a scenario, we have only one receiver on which the SINR values for each link with the other mobile nodes of the network will vary according to their mobility patterns. (iii) Ad hoc mode network, which is the most complex deployment in terms of interference. In this scenario, receivers are spread randomly in the network and the interference levels will be very unstable due to mobility scenarios. All of the nodes have to adapt their rates depending on the channel conditions and network dynamics (node density and mobility). In the second and the third scenarios, all nodes are placed in the communication range of each other to avoid the routing protocol impact and the cases where the receivers become transmitters. This allows for us to concentrate our study on interference impact and mobility impact on RAAs. Each simulation result presented below is the mean value of 50 simulations, the bars in the graphs represent the standard deviation. The values of the network parameters configured in the simulation scripts are summarized in Table 3. A simulation duration of $30 \mathrm{~s}$ is sufficient to ensure randomness in the movement of the nodes inside the boundaries of the square, and it is enough for the selected algorithms to converge. Log-distance and Nakagami-m loss models were used to make the simulations more realistic in terms of link quality and link stability. We used UDP over TCP for traffic generation in order to avoid the overhead of TCP and its rate adaptation which will interfere with the rate adaption of IEEE 802.11. Finally, as for packet size and mobility speed, we decided not to study their impacts in this paper. The chosen values are representative of average to big sized frames and relatively fast moving nodes. 
Table 3. Summary of simulation parameters.

\begin{tabular}{ll}
\hline Parameter & Value \\
\hline Simulation time & $30 \mathrm{~s}$ \\
Runs & 50 \\
WLAN standard & IEEE.802.11ac \\
Path loss model & Log-distance \\
Fast fading loss model & Nakagami-m \\
Traffic & UDP \\
Packet size & 1500 Bytes \\
Data Rate & $60 \mathrm{Mbps}$ (Application Layer) \\
Mobility model & Random Walk 2d Mobility Model \\
Mobility speed & $6 \mathrm{~m} / \mathrm{s}$ \\
Topology size & Square of boundaries $(-100,100,-100,100)$ \\
\hline
\end{tabular}

\subsection{Scenario 1-Interference Free}

In this scenario, we have a simple scenario with only one communication link. We have one access point (AP) and one station. The AP is stationary and the station is moving away at a speed of $6 \mathrm{~m} / \mathrm{s}$ as mentioned in Table 3. The main purpose of this scenario is to evaluate the efficiency of RAAs under the influence of mobility in an interference free network.

We measured the throughput of the four algorithms with the station moving away progressively from the AP. As seen in Figure 1, although the results of all tested algorithms are similar, Ideal and Mutfed RAA performed slightly better than MinstrelHT and Strale. Thanks to its fast feedback and decision making, Ideal algorithm can detect channel changes faster than other algorithms and adapts the rate accordingly. It is noticeable that MutFed has a slightly lower throughput than Ideal due to the fact that MutFed takes the decision every 10 frames. Thus, when the channel conditions deteriorate, the decision is not directly made, causing frame losses. MinstrelHT and Strale have a lower achieved throughput. These two algorithms take some time to lower the rate when needed, because the decision is made based on random probing and FLR in the case of MinstrelHT and on A-MPDU size adaptation in the case of Strale.

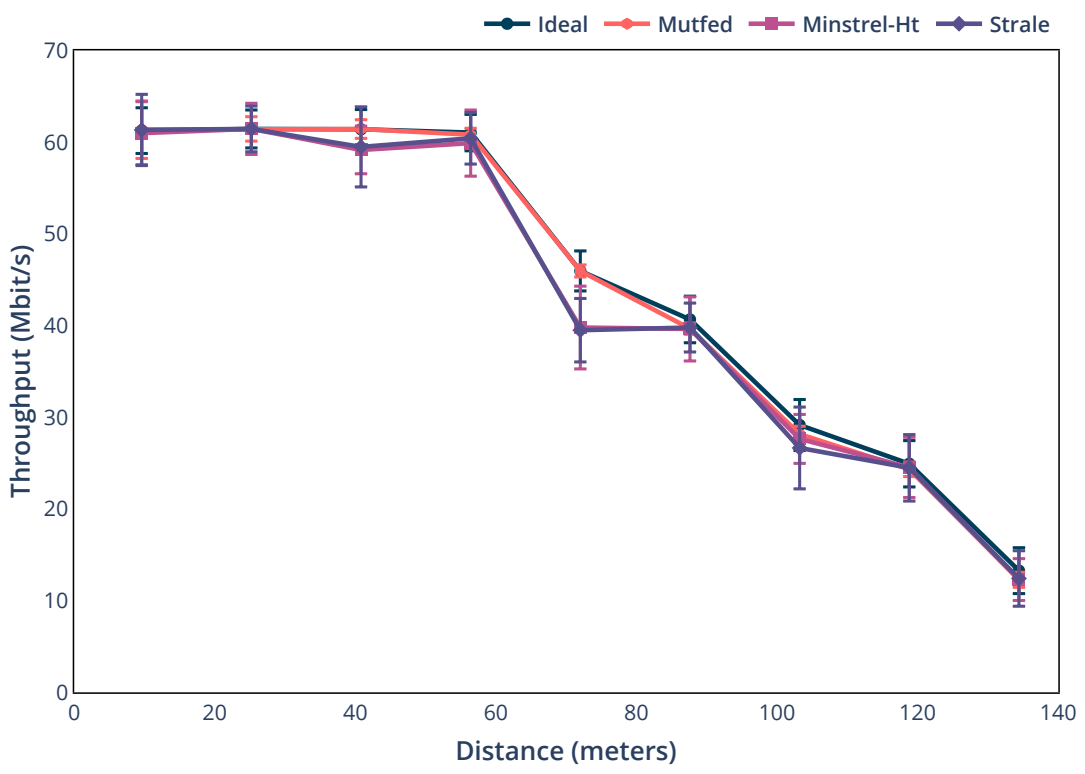

Figure 1. Throughput of a station moving away from an access point at a speed of $6 \mathrm{~m} / \mathrm{s}$. 


\subsection{Scenario 2-Infrastructure Network}

In this scenario, we consider an infrastructure mode network. We have a stationary access point that is placed at the center of the square field and all stations are moving randomly sending constant traffic of $60 \mathrm{Mb} / \mathrm{s}$ to the access point. The number of stations is increased gradually from 5 to 50 .

Figure 2 shows the overall physical throughput received at the access point. Ideal RAA performed better than the other algorithms due to its fast reaction to channel changes, while the two implicit feedback algorithms (Strale and Minstrel-HT) take more time to recover after the channel conditions change. On the other hand, the Strale algorithm performed slightly better than Minstrel-HT, because, instead of decreasing the rate, it tries to find an optimal size of the A-MPDU in which it provides a better throughput at the current rate when compared to stepping to a lower rate.

Figures 3 and 4 show the FLR based on MPDU losses and A-MPDU losses, respectively. We notice high MPDU FLR values, because all of the stations are in the transmission range of each other. This increases the interference level when the node density increases. A-MPDU FLR is a false-positive rate increase in a fast changing channel. It happens when a transmitter decides to increase the rate for the next transmission, but the channel conditions deteriorate before sending the frame. Note that this condition also applies in the MPDU FLR when the channel conditions deteriorates during the transmission of the packet, this can be seen by inspecting the position of the MPDU losses in the BlockAck. Figure 5 shows the average MCS index values selected by the stations. We notice that the stations using the Ideal RAA selected higher MCS index values with almost the same MPDU FLR and lower A-MPDU FLR which explains the higher throughput achieved. All of these frame losses cause MutFed to have the worst performance among the tested algorithms, each 2 frame losses the rate is lowered, causing more channel occupancy and less data being sent.

We noticed from Figure 4 that, when the node density increases ( $>20$ for example) causing the interference level to increase, the gap of the A-MPDU FLR between RAAs relying on SINR and other RAAs is increased. This is due to the fact that the SINR values better reflect the channel condition.

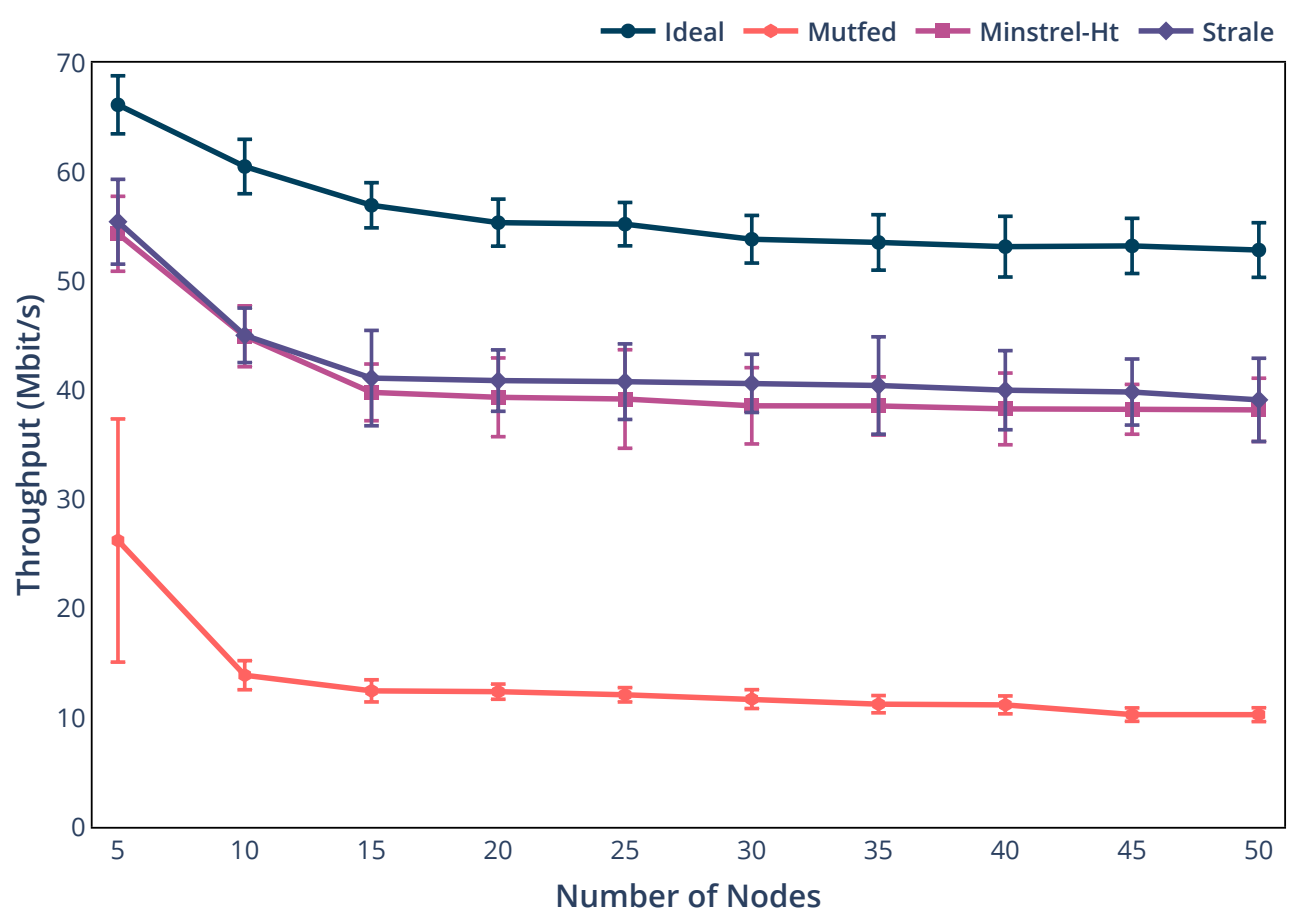

Figure 2. Overall throughput received at the access point. 


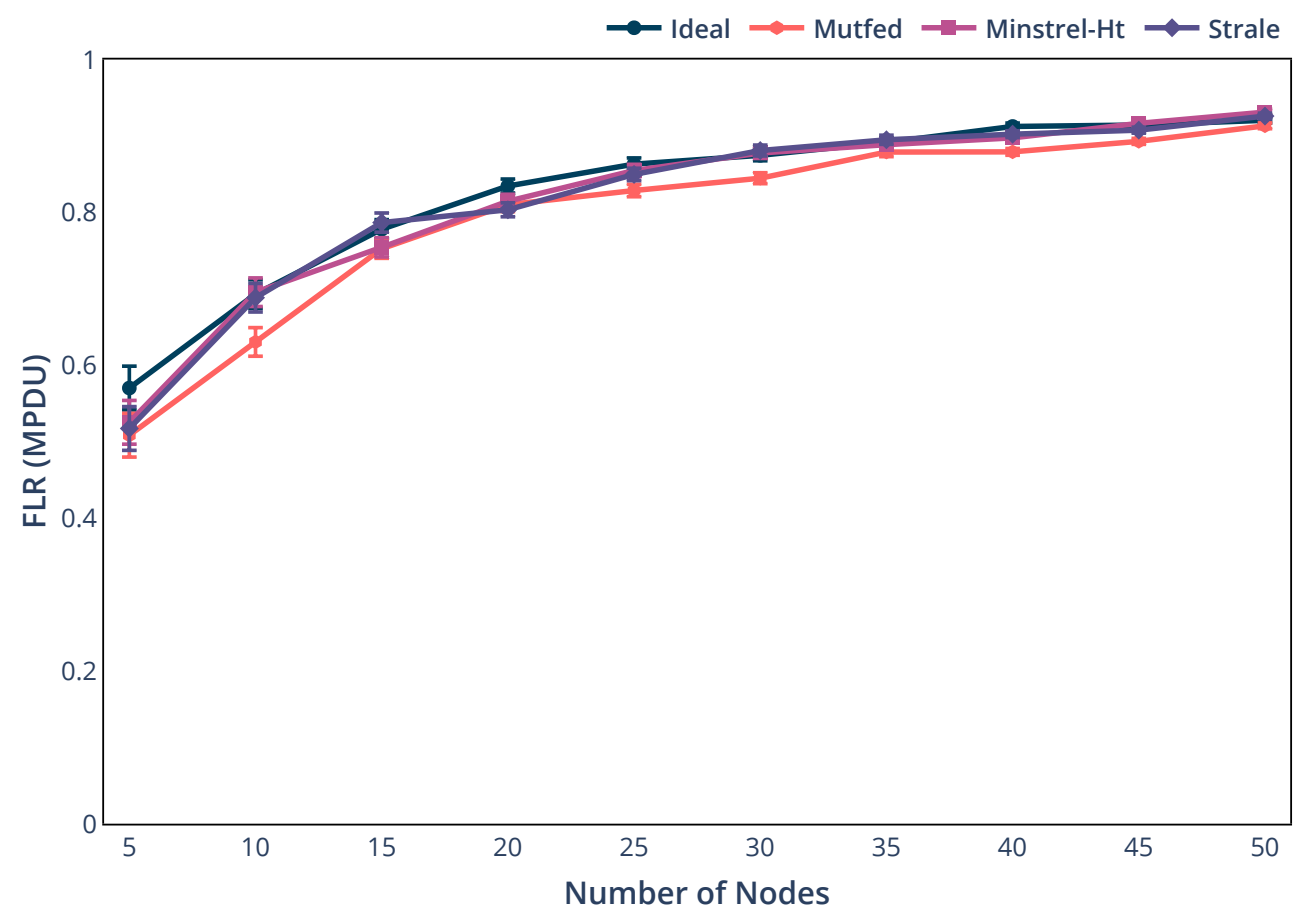

Figure 3. MAC Protocol Data Unit (MPDU) Frame Loss Ratio.

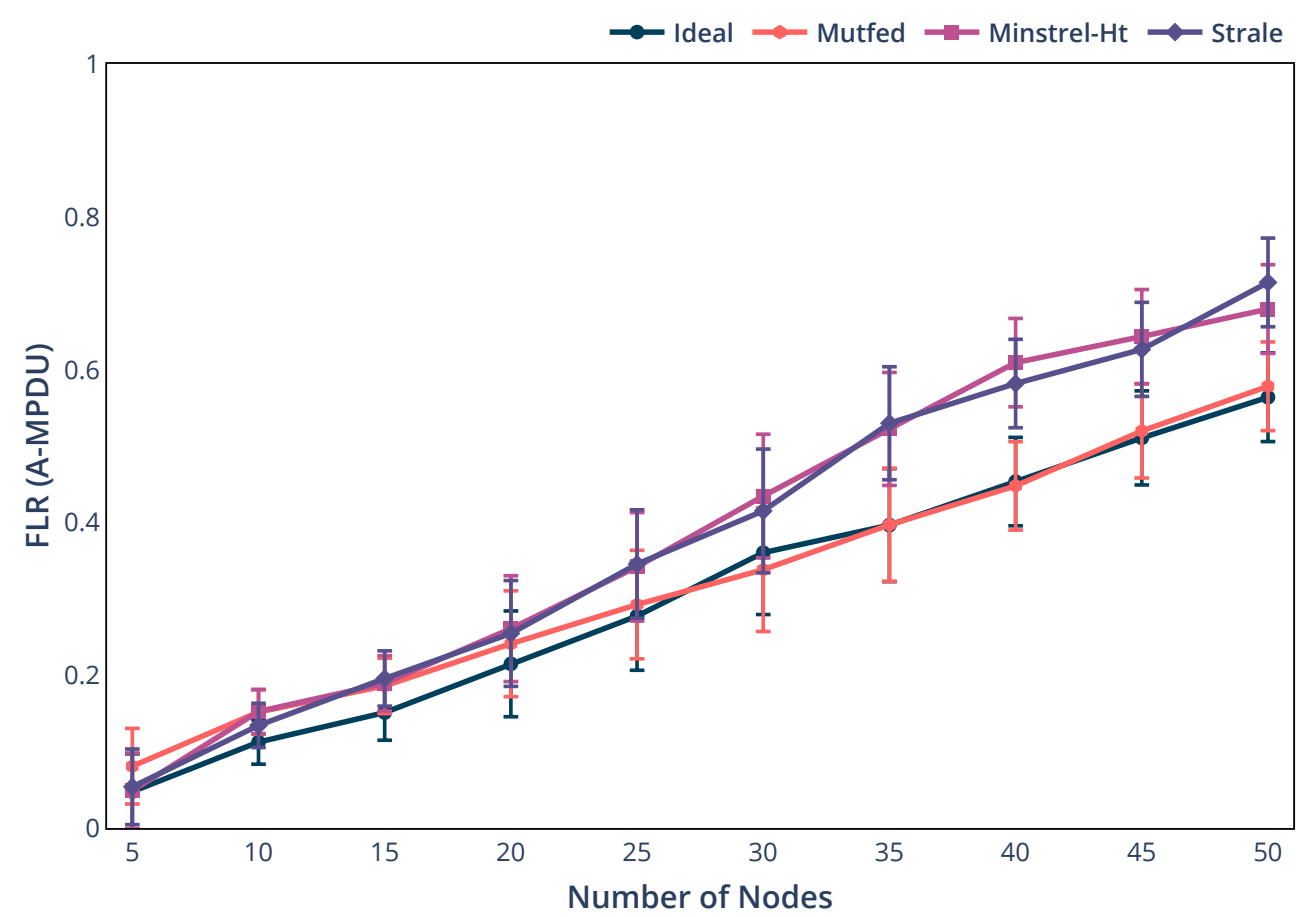

Figure 4. A-MPDU Frame Loss Ratio. 


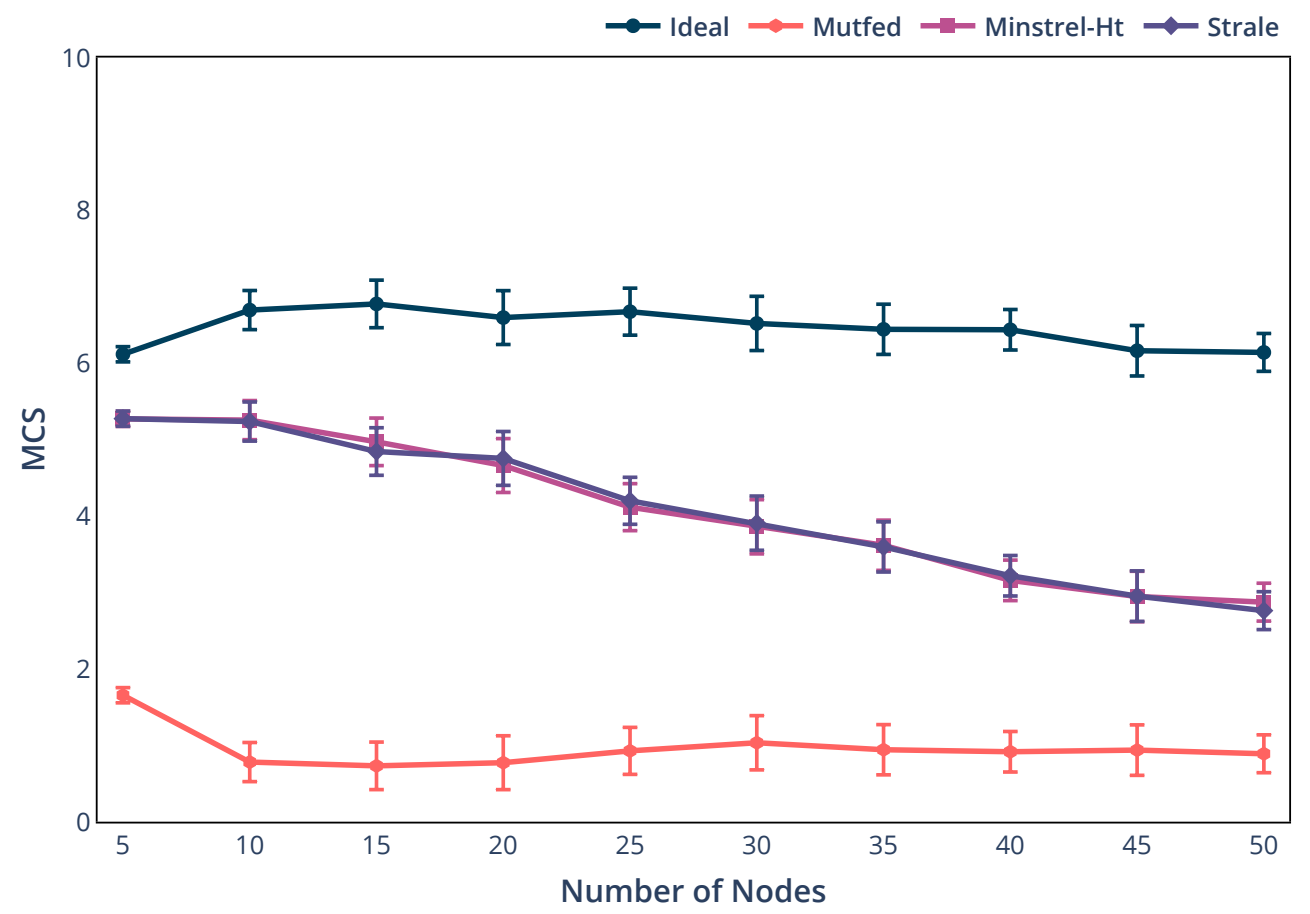

Figure 5. Mean value of the modulation and coding schemes (MCS) used by the nodes.

\subsection{Scenario 3-Ad hoc Network}

In this scenario, we consider an Ad hoc network scenario. All of the nodes are placed randomly in a square field. They move at a speed of $6 \mathrm{~m} / \mathrm{s}$ and change their direction randomly every $3 \mathrm{~s}$. They send a constant UDP traffic rate of $60 \mathrm{Mb} / \mathrm{s}$ to a sink node. Half of the nodes are traffic generators and the other half are sink nodes. The number of nodes is gradually increased from five to 50 nodes.

Figure 6 shows the overall average physical throughput achieved by all sink nodes. Figures 7 and 8 show the FLR based on MPDU losses and A-MPDU losses respectively, combined with the results that are shown in Figure 9 for the selected MCS. It shows that MCS values selected by the stations using Ideal RAA are higher than the ones selected when using other algorithms. The average MCS values in Ad hoc mode are slightly lower than the MCS values in infrastructure mode. This is the reason why the throughput in Ad hoc mode is slightly lower than that of infrastructure mode.

Combined with the FLR, we conclude that the overall throughput of the stations using Ideal RAA is the best among the studied algorithms. In the case of frame losses, the absence of feedback from the receiver prevents the transmitter from adapting the rate according to the changed channel conditions. If the channel conditions deteriorate and frame losses occur, the transmitter will keep sending frames at the same rate, leading to more frame losses until the channel conditions improve.

The false-positive MCS rate decision may cause, in some cases, more A-MPDU frame losses because of channel conditions deterioration before transmitting the new frame, as seen in scenario 2 . 


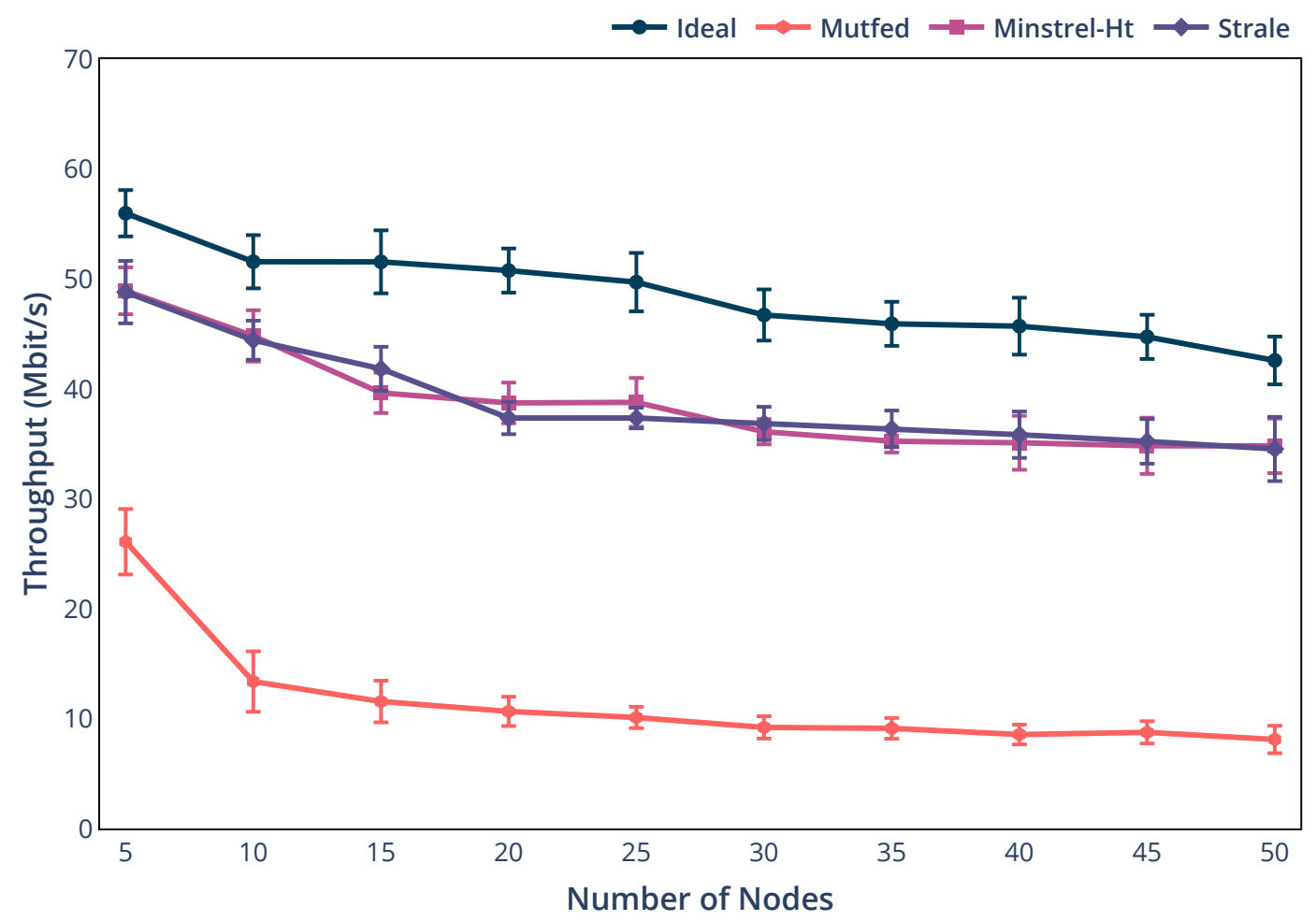

Figure 6. Average throughput of sink nodes in an Ad hoc network.

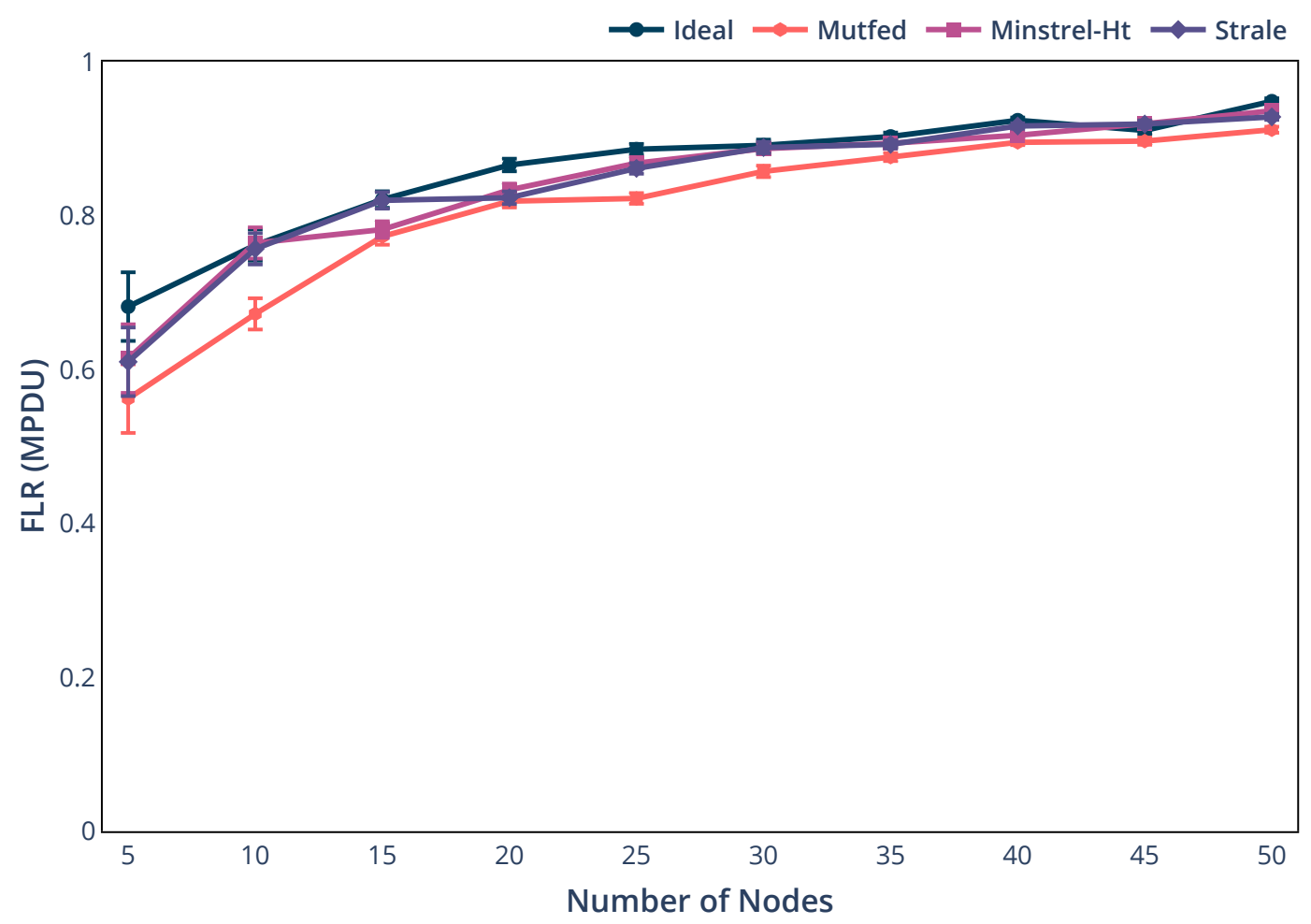

Figure 7. MPDU Frame Loss Ratio. 


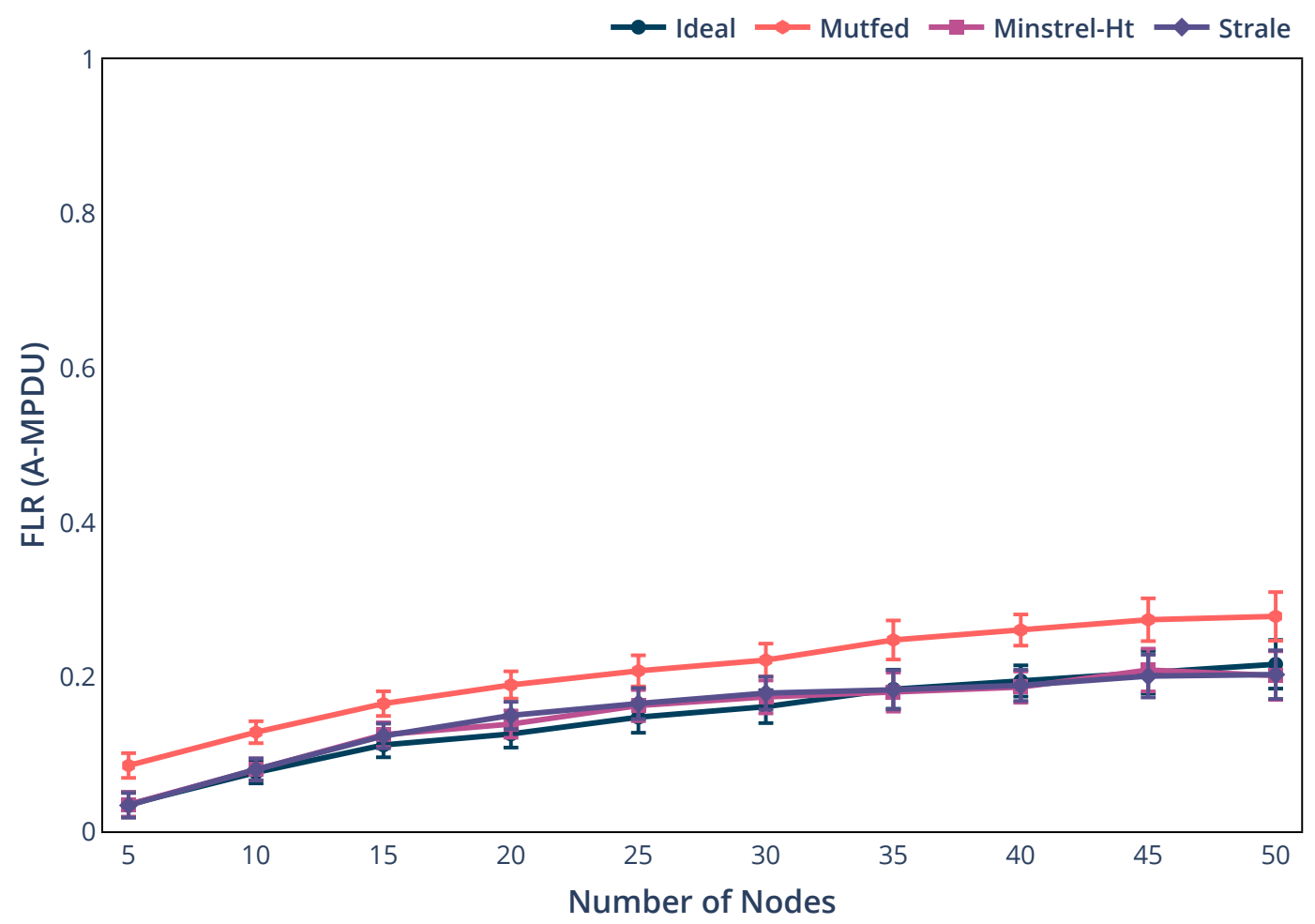

Figure 8. A-MPDU Frame Loss Ratio.

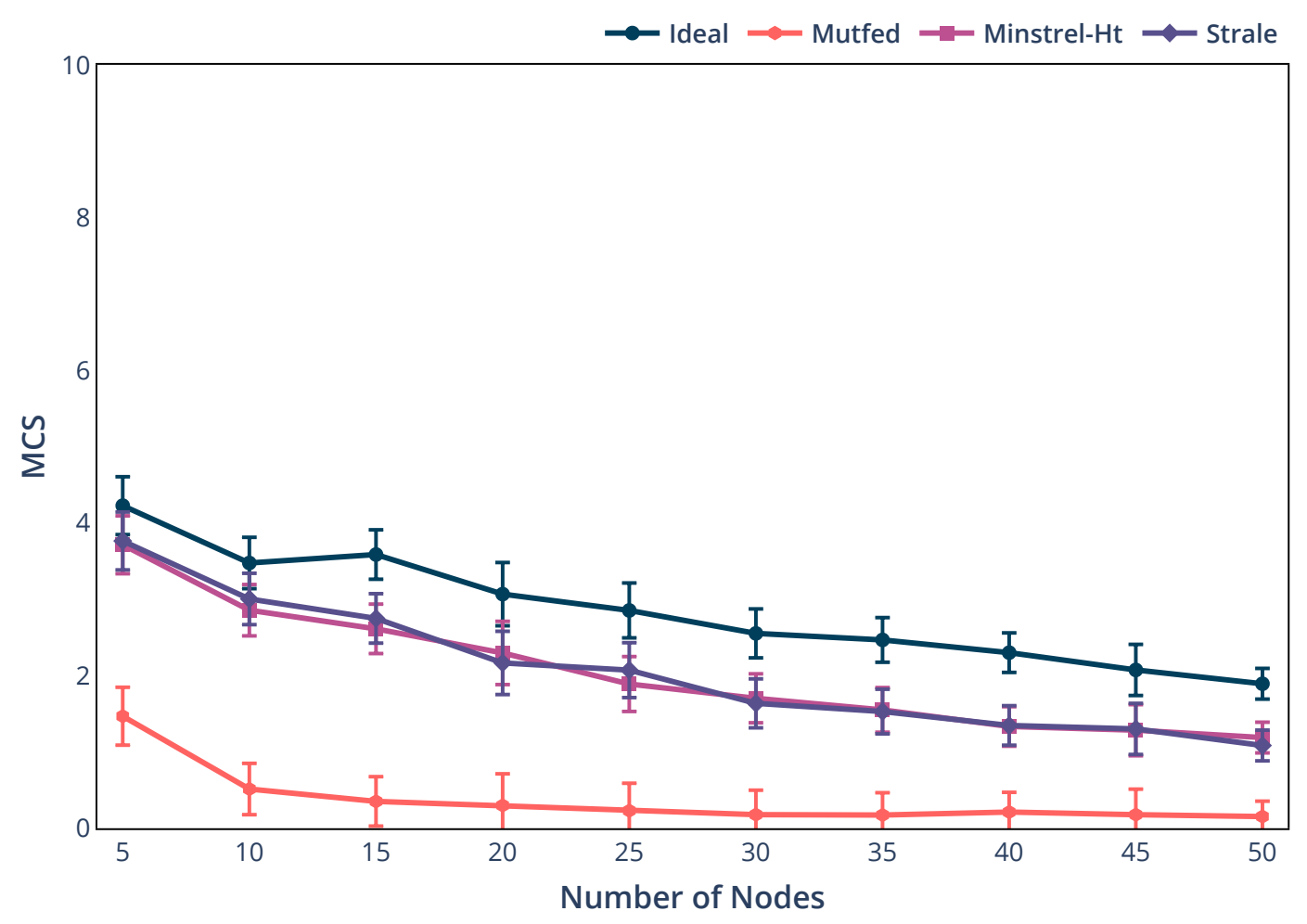

Figure 9. Mean value of the MCS selected by sender nodes. 
Note that, in scenarios 2 and 3, nodes are always in the communication range of each other. This makes the interference level go higher with every new sender node added to the network. This, in turn, explains the high values of FLR. Indeed, FLR based on MPDUs counted in BlockAck reach above $80 \%$ in both scenarios when we have more than 20 nodes in the network. FLR based on A-MPDUs increases gradually with the number of nodes in the network.

\section{Lessons Learned}

In this section, we share the lessons learned from our study of the existing algorithms and the simulation results that open the door for future research ideas.

\subsection{Explicit Feedback}

Most of the explicit feedback approaches rely on physical layer metrics, mainly the SINR, as we have seen in the related work section. Several conditions should be met for this approach to be used on real devices such as a hardware that provides SINR values, or a method for sending the feedback to the transmitter, which does not modify the standard.

Additionally, as simulation results show, only relying on SINR values for decision making may not be enough. In some cases, the absence of the feedback may result in many frame losses until the channel quality is suitable for the current rate. A possible solution to this problem may be implemented on the transmitter side.

Although explicit feedback algorithms, such as Ideal RAA, gave better performances than the other algorithms in a dense environment, in both ad hoc and infrastructure modes, a lot of work needs to be done in order to minimize the FLR, which will result in better overall performances. One can consider different approaches to minimize the FLR by combining the current explicit approach with implicit approaches, such as changing the A-MPDU size, which results in a lower channel occupancy and collecting statistical information at the transmitter that will help in the decision making of future transmissions.

\subsection{Implicit Feedback}

Relying on frame loss ratio and probing rates different than the selected one are the common approaches of the implicit feedback algorithms. Probing random rates should be done very carefully when considering the duration of probing, because choosing a rate that is higher than the selected one may result in many frame losses. Random probing results in slow convergence of the algorithm towards the optimal rate.

Implicit feedback algorithms gave promising results in a mobile collision-free environment, but did not prove to have a better performance than the other categories. This can be improved by trying to detect the degree of mobility and studying the direct effects of mobility on the result of frame transmissions. However, in a dense environment, RAAs should be able to estimate the reason of frame loss and, preferably, should rely on different metrics, which will lead to a more accurate rate decision. Combined with a method for rate changing that ensures increasing the rate to achieve the maximum throughput when channel conditions improve and decreasing the rate when channel conditions deteriorate. This is not an easy task, in order to achieve this, an algorithm should be able to have a real-time estimation of the conditions, such as SINR.

\subsection{Hybrid Approach}

Simulation results showed that, in a dense environment, decreasing the rate after failed transmissions leads to longer transmission times and a decrease in the throughput, which, in turn, increases the occupation time of the channel affecting all of the nodes in the network. One of the possible approaches to fix this may be using a sliding window that will help predict the future channel conditions and act accordingly [24]. 
Designing an efficient hybrid approach is a challenging task on which few existing works have concentrated. The simulation results showed that the selected hybrid algorithm (namely Mutfed) gave worse performances than the other algorithms, this is mainly due to the way Mutfed decreases its bit rate, which, in turn, decreases throughput and increases interference and frame loss. Ideally, a hybrid algorithm could be constructed on the top of an explicit algorithm by including additional metrics, such as collecting statistics about FLR that can be used to adapt the number of MPDUs in an A-MPDU.

\section{Conclusions}

Rate adaptation algorithms help wireless networks achieve better performance by selecting the most suitable MCS, depending on the channel state. These algorithms are rarely specified in the wireless standards and they are left for the constructors to implement. In this paper, we have presented several rate adaptation algorithms and grouped them into different categories according to the way they function. We also provided an analysis for these algorithms highlighting their functionalities and drawbacks. We implemented algorithms of different categories in the NS-3 simulator and performed a comparison study for Ad hoc and infrastructure modes.

Our study consisted of evaluating the impact of interference and mobility on RAAs in different scenarios. We analyzed several metrics, such as Throughput, Frame Loss Ratio, and selected MCS. The simulation results show that using metrics that give a good indication of the channel conditions such as SINR and reacting instantaneously, which is the case of the Ideal RAA, lead to selecting the rate which provides the best achievable throughput. Otherwise, relying on frame loss ratio or making rate decisions without distinguishing the cause of frame losses lead to a slow process to reach the best rate.

Analysis of simulation results shows that we need to be able to estimate the channel state to improve the delivery success of the next transmission in order to have a robust RAA. This is achieved by selecting an MCS that maximizes the throughput under the current channel state. In addition, having a process that predicts future channel state may help preventing frame losses.

A lot of research has been done on rate adaptation algorithms addressing different scenarios and environments, relying on a variety of metrics, only few RAAs have been evaluated on real devices. Hence, the field is still open to many possible improvements. In our future works, we will work on the enhancements that we proposed in Section 4, namely extending Ideal RAA by adding implicit metrics for better decision making. We will also study the impact of routing algorithms on the overall performance of the network in Ad hoc scenarios. In this paper, we focused on dense deployment and high mobility in infrastructure and ad hoc modes; in our future works, we will also study the impact of mobility scenarios and application profiles in terms of data rate and packet size on the performance of RAAs.

Author Contributions: Conceptualization, I.S. and G.C.; methodology, I.S. and G.C.; software, I.S.; validation, I.S. and G.C.; investigation, I.S. and G.C.; resources, I.S. and G.C.; data curation, I.S.; writing-original draft preparation, I.S. and G.C.; writing-review and editing, I.S. and G.C.; visualization, I.S.; supervision, G.C.; project administration, G.C.; funding acquisition, G.C. All authors have read and agreed to the published version of the manuscript.

Funding: This research was funded by the French government IDEX-ISITE initiative 16-IDEX-0001 (CAP 20-25).

Conflicts of Interest: The authors declare no conflict of interest.

\section{References}

1. Bhover, S.U.; Tugashetti, A.; Rashinkar, P. V2X communication protocol in VANET for co-operative intelligent transportation system. In Proceedings of the 2017 International Conference on Innovative Mechanisms for Industry Applications (ICIMIA), Bangalore, India, 21-23 February 2017; pp. 602-607. [CrossRef]

2. Yang, W.; Wang, X.; Song, X.; Yang, Y.; Patnaik, S. Design of Intelligent Transportation System Supported by New Generation Wireless Communication Technology. Int. J. Ambient. Comput. Intell. 2018, 9, 78-94. [CrossRef] 
3. Jawad, H.M.; Nordin, R.; Gharghan, S.K.; Jawad, A.M.; Ismail, M. Energy-Efficient Wireless Sensor Networks for Precision Agriculture: A Review. Sensors 2017, 17, 1781. [CrossRef] [PubMed]

4. Kulatunga, C.; Shalloo, L.; Donnelly, W.; Robson, E.; Ivanov, S. Opportunistic Wireless Networking for Smart Dairy Farming. IT Prof. 2017, 19, 16-23. [CrossRef]

5. Yao, Y.; Chen, X.; Rao, L.; Liu, X.; Zhou, X. LORA: Loss Differentiation Rate Adaptation Scheme for Vehicle-to-Vehicle Safety Communications. IEEE Trans. Veh. Technol. 2017, 66, 2499-2512. [CrossRef]

6. Wu, Y.; Yu, W.; Griffith, D.; Golmie, N. Modeling and Performance Assessment of Dynamic Rate Adaptation for M2M Communications. IEEE Trans. Netw. Sci. Eng. 2020, 7, 285-303. [CrossRef]

7. He, S.; Wang, W.; Yang, H.; Cao, Y.; Jiang, T.; Zhang, Q. State-Aware Rate Adaptation for UAVs by Incorporating On-Board Sensors. IEEE Trans. Veh. Technol. 2020, 69, 488-496. [CrossRef]

8. Yofune, M.; Suto, M.; Amezawa, Y.; Sato, S. Rate-adaptation scheme for multiband WLAN system. In Proceedings of the 2018 Advances in Wireless and Optical Communications (RTUWO), Riga, Latvia, 15-16 November 2018; pp. 72-77. [CrossRef]

9. Biaz, S.; Wu, S. Rate adaptation algorithms for IEEE 802.11 networks: A survey and comparison. In Proceedings of the 2008 IEEE Symposium on Computers and Communications, Marrakech, Morocco, 6-9 July 2008; pp. 130-136. [CrossRef]

10. Yin, W.; Hu, P.; Indulska, J.; Portmann, M.; Mao, Y. MAC-layer rate control for 802.11 networks: A survey. Wirel. Netw. 2020, 26, 3793-3830. [CrossRef]

11. Schmidt, F.; Hithnawi, A.; Punal, O.; Gross, J.; Wehrle, K. A receiver-based 802.11 rate adaptation scheme with on-demand feedback. In Proceedings of the 2012 IEEE 23rd International Symposium on Personal, Indoor and Mobile Radio Communications (PIMRC), Sydney, NSW, Australia, 9-12 September 2012; pp. 399-405.

12. Lee, O.; Kim, J.; Lim, J.; Choi, S. SIRA: SINR-Aware intra-frame rate adaptation. IEEE Commun. Lett. 2015, 19, 90-93. [CrossRef]

13. Arif, T.Y.; Munadi, R. Evaluation of the minstrel-HT rate adaptation algorithm in IEEE 802.11n WLANs. Int. J. Simul. Syst. Sci. Technol. 2017, 18, 11.1-11.7. [CrossRef]

14. Byeon, S.; Yoon, K.; Yang, C.; Choi, S. STRALE: Mobility-aware PHY rate and frame aggregation length adaptation in WLANs. In Proceedings of the IEEE INFOCOM 2017-IEEE Conference on Computer Communications, Atlanta, GA, USA, 1-4 May 2017.

15. Felix, F. Minstrel ht: New Rate Control Module for 802.11n. Available online: http://1wn.net/Articles/ 376765/ (accessed on 19 April 2020).

16. Pefkianakis, I.; Lee, S.B.; Lu, S. Towards MIMOAware 802.11n Rate Adaptation. IEEE/ACM Trans. Netw. 2013, 21, 692-705. [CrossRef]

17. Nguyen, D.; Garcia-Luna-Aceves, J.J. A practical approach to rate adaptation for multi-antenna systems. In Proceedings of the 2011 19th IEEE International Conference on Network Protocols, Vancouver, BC, Canada, 17-20 October 2011; pp. 331-340.

18. Kriara, L.; Marina, M.K. SampleLite: A Hybrid Approach to 802.11n Link Adaptation. ACM SIGCOMM Comput. Commun. Rev. 2015, 45, 4-13. [CrossRef]

19. Selinis, I.; Katsaros, K.; Vahid, S.; Tafazolli, R. Damysus: A Practical IEEE 802.11ax BSS Color Aware Rate Control Algorithm. Int. J. Wirel. Inf. Netw. 2019, 26, 285-307. [CrossRef]

20. Yin, W.; Hu, P.; Indulska, J.; Bialkowski, K. Performance of mac80211 rate control mechanisms. In Proceedings of the 14th ACM International Conference on Modeling, Analysis and Simulation of Wireless and Mobile Systems, Miami, FL, USA, 31 October-4 November 2011; pp. 427-436. [CrossRef]

21. Khan, S.; Mahmud, S.A.; Noureddine, H.; Al-Raweshidy, H.S. Rate-adaptation for multi-rate IEEE 802.11 WLANs using mutual feedback between transmitter and receiver. In Proceedings of the PIMRC, Instanbul, Turkey, 26-30 September 2010; pp. 1372-1377.

22. Huang, T.; Chen, H.; Zhang, Z.; Cui, L. EasiRA: A hybrid rate adaptation scheme for 802.11 mobile wireless access networks. In Proceedings of the IEEE Wireless Communications and Networking Conference, WCNC, Paris, France, 1-4 April 2012; pp. 1520-1525. [CrossRef] 
23. Available online: https:/ / wireless.wiki.kernel.org/en/developers/documentation/mac80211/ratecontrol/ minstrel (accessed on 19 April 2020).

24. Judd, G.; Wang, X.; Steenkiste, P. Efficient channel-aware rate adaptation in dynamic environments. In Proceedings of the 6th International Conference on Mobile Systems, Applications, and Services, Breckenridge, CO, USA, 17-20 June 2008; pp. 118-131. [CrossRef]

(C) 2020 by the authors. Licensee MDPI, Basel, Switzerland. This article is an open access article distributed under the terms and conditions of the Creative Commons Attribution (CC BY) license (http:/ / creativecommons.org/licenses/by/4.0/). 